\title{
The atomic gas in outer disks in semi-analytic models of galaxy formation $\dagger$
}

\author{
Jian $\mathrm{Fu}^{1}$ and $\mathrm{Yu} \mathrm{Luo}^{2}$ \\ ${ }^{1}$ Key Laboratory for Research in Galaxies and Cosmology, Shanghai Astronomical \\ Observatory, CAS, 80 Nandan Road., Shanghai, 200030, China, \\ email: fujian@shao.ac.cn \\ ${ }^{2}$ Purple Mountain Observatory, 2 West Beijing Road, Nanjing, 210008, China
}

\begin{abstract}
We use semi-analytic models of galaxy formation L-Galaxies based on $\Lambda$ CDM cosmology to study the HI gas component in galaxy outskirts. We adopt the radially-resolved version of the models by $\mathrm{Fu}$ et al. (2013), which includes both atomic and molecular gas component in interstellar medium. This model has been recently updated by Luo et al. (2016) to include cold gas stripping in the outer disk regions of the satellite galaxies by ram pressure. In our models, we can perfectly reproduce the HI size-mass relation, which is discovered by Broeils \& Rhee (1997) and confirmed by many subsequent observations. In our model, the reason for such tight correlation between HI size and mass is atomic-molecular phase conversion in high gas surface density regions while HI ionization in low gas surface density region, which leads to very narrow distribution of $\mathrm{HI}$ mean surface density. The models also reproduce the universal exponential HI radial profiles in galaxy outskirts found by Bluedisk (Wang et al. 2013), which arises from cold gas accretion onto the galaxy disks in exponentially profiles.
\end{abstract}

Keywords. galaxies: formation, galaxies: ISM, ISM: atoms
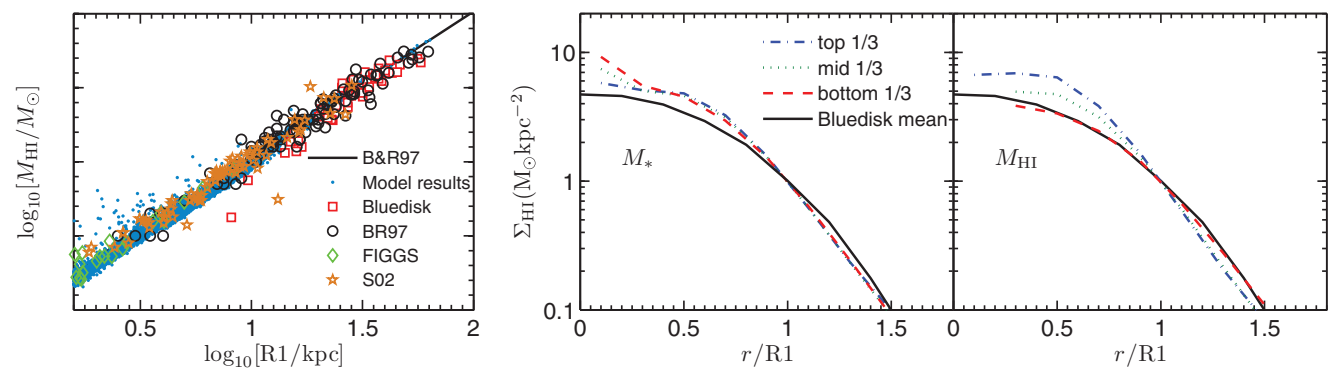

Figure 1. Left panel: The size-mass relation of HI gas in disk galaxies. The blue dots show the model results at $z=0$, and colour dots show various observations for comparison. Right two panels: The universal exponential HI surface density profiles in outskirts of galaxy disks. The model galaxies are divided into three sub-samples according to the stellar mass and HI gas mass. For comparison, the black curves are the observational results from Bluedisk.

\section{References}

Fu, J., Kauffmann, G., Huang, M.-L., et al. 2013, MNRAS, 434, 1531

Luo, Y., Kang, X., Kauffmann, G., \& Fu, J. 2016, MNRAS, 458, 366

Wang, J., Fu, J., Aumer, M., et al. 2014, MNRAS, 441, 2159

$\dagger$ Supported by Natural Science Foundation of China (No.11303072, U1531123). 